\title{
Evaluation of Equipment Maintenance Support Ability Based on Binary Semantics
}

\author{
Xianming Shi a, Dunxiang Zhu, Xiaobo Su, Yongle Wu \\ Army Engineering University, shijiazhuang050003 China. \\ ashixianming@163.com
}

Keywords: Binary semantics; equipment maintenance support; ability assessment.

\begin{abstract}
In order to effectively overcome the original evaluation method of subjectivity, the complexity of equipment maintenance, the uncertainty problem, based on the analysis of existing evaluation method, construct the evaluation index system of equipment maintenance support capability, illustrates the basic principle of two tuple, will evaluate the two tuple and capacity closely, given the equipment maintenance support capability two Yuan based on semantic evaluation method, and analyzes an example. Finally, we compare the two-tuple linguistic analysis with AHP and BP neural network. The results show that the method can objectively evaluate the equipment maintenance support ability and has certain reference value.
\end{abstract}

\section{Preface}

Equipment maintenance support capability refers to the ability to maintain and restore the equipment to maintain or restore the prescribed technical status or improve the equipment's capabilities in the stage of use of all levels of maintenance and security agencies within the military's military and military establishment system after equipment is dispatched to the military. As an important part of life-long system-wide management, the assessment of equipment maintenance support capabilities facilitates an intuitive understanding of existing maintenance support capabilities, exposes problems in equipment maintenance support, and finds weak links to guide the future construction of equipment maintenance support. It is of great practical significance to improve equipment maintenance and safeguard the environment and continuously improve equipment maintenance support capabilities.

In the evaluation of equipment maintenance support capabilities, some scholars have put forward many methods. At present, the research on equipment maintenance support capability evaluation mainly focuses on two aspects: the construction of the evaluation index system and the development of the evaluation algorithm model. In the evaluation index system construction, Yao Shilling [1] started with the concept of equipment maintenance support capabilities and evaluation principles, analyzed the constituent elements of the armored equipment maintenance support capability system, and constructed an evaluation index system for basic maintenance support capabilities of armored equipment; $\mathrm{Wu}$ Jian gang [2] from To establish the guiding ideology of the index system of wartime equipment maintenance support capabilities, to assess the maintenance support capabilities and maintenance effects in a concise manner, and to give the corresponding calculation formula; Shad Xinyang [3] and others learn from the "competency model". Combine the special operations with the actual situation to build a "task-function" model. In the development of the evaluation algorithm model, Zhang Xiao Hua [4] established a corresponding evaluation index system for the basic problems of the maintenance of Sky wave Radar, explained in detail the intrinsic meanings and calculation formulas of the indicators, and finally used the analytic hierarchy process in combination with the actual data for maintenance. The protection capability was calculated; Zhang Lei [5] used simulation methods to propose an evaluation method for equipment maintenance support capability based on Extends simulation and combined neural network. Finally, the application verification was carried out with a certain equipment maintenance support system. However, due to the complexity and uncertainty of equipment maintenance support, existing methods such as AHP, neural networks, 
and set-the-pair methods are often subjective, so evaluations of equipment maintenance support indicators are often not as accurate and often require using fuzzy information to evaluate the index system.

Based on this, this paper analyzes the weaknesses of existing assessment methods and proposes a method for evaluating equipment maintenance support capabilities based on binary semantics. It uses fuzzy information to evaluate evaluation indicators and improves the accuracy and objectivity of equipment maintenance support capability assessment., And with the example analysis to verify the feasibility of the method, it is easy to provide decision-making basis for improving the future maintenance work.

\section{Construction of Equipment Maintenance Support Ability Evaluation Index System}

The establishment of equipment maintenance support capability evaluation index system should not only follow the principles of systematicity, orientation, testability, independence, and operability, but also comprehensively consider the specificity of equipment maintenance support [8]. This article analyzes five aspects including the protection of mobility, equipment repairs capabilities, maintenance management capabilities, resource support capabilities, and social security capabilities.

To sum up, we analyzed five aspects such as the protection of mobility, equipment repairs capabilities, maintenance management capabilities, resource support capabilities, and social security capabilities, and established an index system for equipment maintenance support capabilities, as shown in Figure 1.

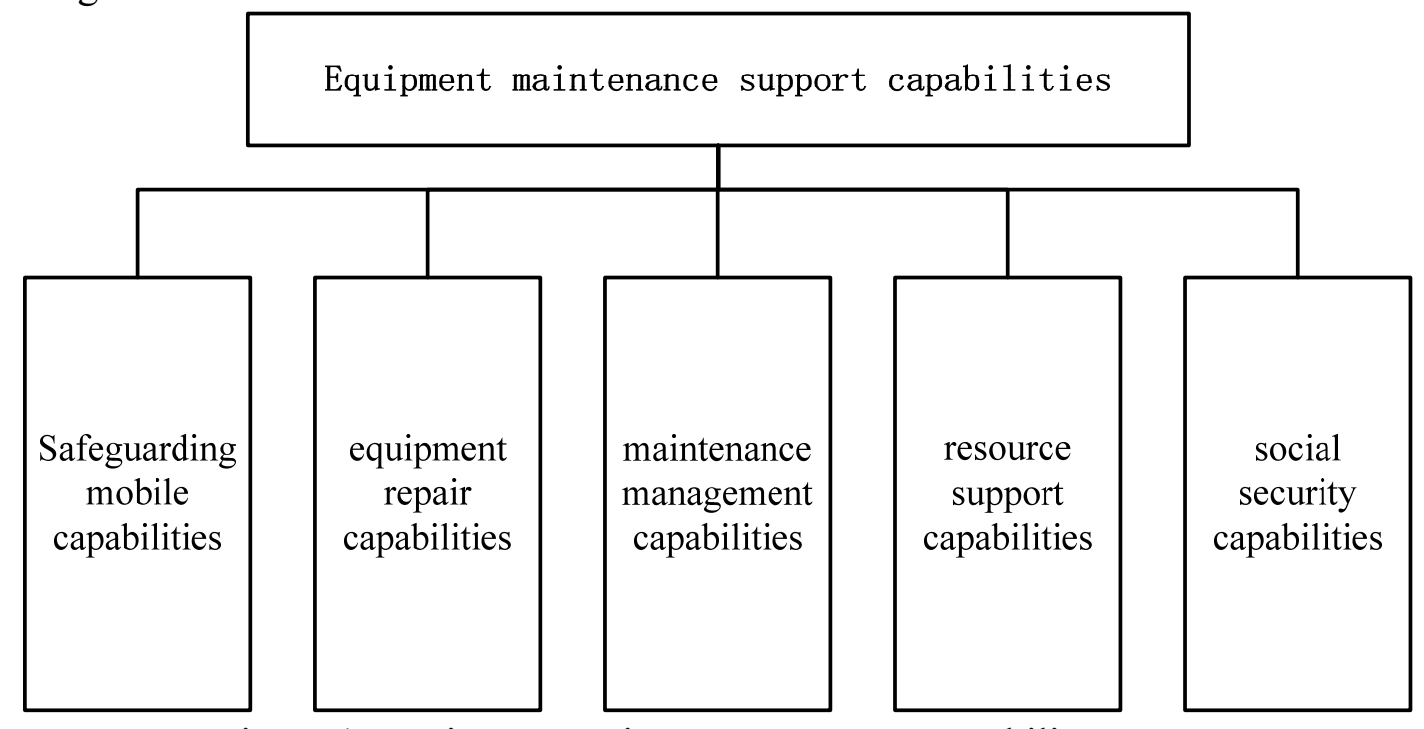

Figure 1. Equipment maintenance support capability system

\section{Binary Semantics}

Binary semantic information refers to the use of a binary group $\left(s_{k}, a_{k}\right)$ to represent the language evaluation information given by the expert for the evaluation index, and $s_{k}$ represents the first element of a previously defined language evaluation set, which is the difference between $s_{k}$ and the symbol transfer value, the representation and the evaluation result, And meet $a_{k} \in[-0.5,0.5]$.

Definition 1: Assume that $S$ for a language phrase, ${ }^{s_{k}} \in S$ the corresponding binary semantics can be obtained by converting the function according to Equation (1):

$$
\theta: S \rightarrow S \times[-0.5,0.5] \quad \theta\left(s_{k}\right)=\left(s_{i}, 0\right), s_{i} \in S
$$

Definition 2: Assuming that real numbers are used to represent $\beta, \beta \in[0, T]$ linguistic evaluation sets are represented by $S=\left\{s_{0}, s_{1}, \cdots, s_{T}\right\}$, representing the result of a set of language evaluation set 
aggregation operations, and its corresponding binary semantic form can be represented by a function $\Delta$ :

Which is:

$$
\Delta(\beta)=\left(s_{k}, a_{k}\right)=\left\{\begin{array}{c}
s_{k}, \operatorname{round}(\beta) \\
a_{k}=\beta-k, a_{k} \in[-0.5,0.5]
\end{array}\right.
$$

Among them, round is "rounded off" rounding operator.

On the contrary, if it is binary semantics, you can use the inverse function $\Delta^{-1}$ to get the corresponding $\beta \in[0, T]$.which is:

$$
\Delta^{-1}\left(s_{k}, a_{k}\right)=\mathrm{k}+a_{k}=\beta
$$

Definition 3: If $\left\{\left(s_{1}, a_{1}\right),\left(s_{2}, a_{2}\right), \cdots,\left(s_{n}, a_{n}\right)\right\}$ is a set of binary semantics, then the set of binary semantic arithmetic mean operators can be defined as:

$$
(\bar{s}, \bar{a})=\Delta\left(\frac{\sum_{i=1}^{n} \Delta^{-1}\left(s_{i}, a_{i}\right)}{n}\right) \quad \tilde{s} \in S, \tilde{a} \in[-0.5,0.5]
$$

Definition 4: If there is a set of binary semantics $\left\{\left(s_{1}, a_{1}\right),\left(s_{2}, a_{2}\right), \cdots,\left(s_{n}, a_{n}\right)\right\}$ whose corresponding binary semantic information weight vector is $\mathrm{W}=\left\{\left(w_{1}, \gamma_{1}\right),\left(w_{2}, \gamma_{2}\right), \cdots,\left(w_{i}, \gamma_{i}\right)\right\}$, then equation (5) can be used to define a weighted arithmetic mean operator based on binary semantic information:

$$
(\tilde{s}, \tilde{a})=\Delta\left(\frac{\sum_{i=1}^{n} \Delta^{-1}\left(\mathbb{w}_{i}, \gamma_{i}\right) \times \Delta^{-1}\left(s_{i}, a_{i}\right)}{\sum_{i=1}^{n} \Delta^{-1}\left(w_{i}, \gamma_{i}\right)}\right) \tilde{s} \in S, \tilde{a} \in[-0.5,0.5]
$$

\section{Research of Maintenance Support Ability Assessment Method Based on Dual Semantics Equipment}

When assessing equipment maintenance support capabilities, the major problem is that the evaluation value given by experts is not accurate enough due to the ambiguity of the evaluation indicators. The use of linguistic variables can eliminate the ambiguity of this data as much as possible, making the results more reasonable. This paper introduces the binary semantic information into the evaluation of equipment maintenance support capabilities. Let $\mathrm{Me}$ be the collection of experts $E=\left(\mathrm{e}_{1}, \mathrm{e}_{2}, \cdots, \mathrm{e}_{m}\right)$, set $A$ as $A=\left(a_{1}, a_{2}, \cdots, a_{n}\right)$, set $S$ the index evaluation set, and write it as the evaluation language set, $=\{=\mathrm{VG}($ very good $),=\mathrm{G}($ good $),=\mathrm{F}$ (general), $=\mathrm{P}($ difference $),=\mathrm{VP}($ very poor $)\}$, for weighted language sets, $=\{=\mathrm{VI}$ (very important), $=\mathrm{I}$ (important), $=\mathrm{F}$ (general),$=\mathrm{U}$ (not important), $=\mathrm{VU}$ (don't care) $\}$.

The specific steps are:

1) For the $k$ maintenance and repair unit, the $i$ expert gives the corresponding language evaluation value to the first evaluation indicator, and then the formula (11) can be used to convert the language evaluation value into a binary matrix $\left(s_{k}^{j}, 0\right)$.

2) The experts $i$ shall give the weights of language evaluations of the maintenance and protection units on each evaluation index and convert the weighted language evaluation values into a binary matrix $\left(w_{i}^{j}, 0\right)$ by Equation (11).

3) Assemble the binary weights given by the evaluators and use Equation (13) to obtain the arithmetic mean binary semantic weight $\left(\bar{w}_{j}, \bar{a}_{j}\right)$ for each evaluation index.

4) Through the calculation of the previous step, using formula (15) to aggregate the evaluation indicators given by each expert, thus obtaining the binary semantic evaluation results, and finally get the binary semantic evaluation value of the weighted arithmetic mean $R_{i}$.

5) Use formula (14) to aggregate ${ }^{R_{i}}$ and calculate the comprehensive information of the binary semantics obtained by the expert evaluation.

6) Analysis and summary of evaluation results. 


\section{Example Analysis}

Taking an established maintenance and repair unit as an example, we first invite three experts in related fields, and $\mathrm{E}$ is a collection of experts, $E=\left(\mathrm{e}_{1}, \mathrm{e}_{2}, \cdots, \mathrm{e}_{m}\right)$ that is, $\mathrm{m}=3$, and make a corresponding language assessment of the 16 evaluation indicators and their corresponding weights. The index evaluation set is A., denoted as $A=\left(a_{1}, a_{2}, \cdots, a_{n}\right), \mathrm{n}=16$, the results are given in Table 1 and Table 2, respectively. Specific steps are as follows:

(1) Convert the linguistic value in Table 1 into a binary matrix. The results after processing are shown in Table 3.

(2) Convert the weighted language evaluation values of the evaluation indexes in Table 2 into a binary matrix. The results are shown in Table 4.

Table 1. Equipment maintenance assurance indicators language evaluation results

\begin{tabular}{|c|c|c|c|c|c|c|c|c|c|c|c|c|c|c|c|c|}
\hline & $a_{1}$ & $a_{2}$ & $a_{3}$ & $a_{4}$ & $a_{5}$ & $a_{6}$ & $a_{7}$ & $a_{8}$ & $a_{9}$ & $a_{10}$ & $a_{11}$ & $a_{12}$ & $a_{13}$ & $a_{14}$ & $a_{15}$ & $a_{16}$ \\
\hline $\mathrm{e}_{1}$ & $\mathrm{P}$ & $\mathrm{F}$ & $\mathrm{F}$ & $\mathrm{F}$ & VP & $\mathrm{P}$ & $\mathrm{F}$ & VP & VP & $\mathrm{F}$ & VP & $\mathrm{P}$ & $\mathrm{P}$ & $P$ & $\mathrm{P}$ & $\mathrm{F}$ \\
\hline$e_{2}$ & VP & $\mathrm{F}$ & VP & VP & $\mathrm{P}$ & $\mathrm{F}$ & VP & $\mathrm{F}$ & $\mathrm{P}$ & VP & $\mathrm{F}$ & $\mathrm{F}$ & VP & $\mathrm{F}$ & $\mathrm{F}$ & $\mathrm{F}$ \\
\hline $\mathrm{e}_{3}$ & VG & VP & $\mathrm{G}$ & VG & VG & $\mathrm{F}$ & VG & $\mathrm{G}$ & $\mathrm{G}$ & VG & VP & $\mathrm{G}$ & $\mathrm{G}$ & $\mathrm{P}$ & $\mathrm{F}$ & VP \\
\hline
\end{tabular}

Table 2. Evaluation results of equipment maintenance support evaluation index weight

\begin{tabular}{|c|c|c|c|c|c|c|c|c|c|c|c|c|c|c|c|c|}
\hline & $a_{1}$ & $a_{2}$ & $a_{3}$ & $a_{4}$ & $a_{5}$ & $a_{6}$ & $a_{7}$ & $a_{8}$ & $a_{9}$ & $a_{10}$ & $a_{11}$ & $a_{12}$ & $a_{13}$ & $a_{14}$ & $a_{15}$ & $a_{16}$ \\
\hline $\mathrm{e}_{1}$ & $\mathrm{~F}$ & $\mathrm{I}$ & $\mathrm{I}$ & $\mathrm{VI}$ & $\mathrm{I}$ & $\mathrm{I}$ & $\mathrm{VI}$ & $\mathrm{U}$ & $\mathrm{I}$ & $\mathrm{F}$ & $\mathrm{I}$ & $\mathrm{I}$ & $\mathrm{F}$ & $\mathrm{I}$ & $\mathrm{I}$ & $\mathrm{I}$ \\
\hline $\mathrm{e}_{2}$ & $\mathrm{VI}$ & $\mathrm{F}$ & $\mathrm{U}$ & $\mathrm{VU}$ & $\mathrm{F}$ & $\mathrm{F}$ & $\mathrm{I}$ & $\mathrm{VI}$ & $\mathrm{I}$ & $\mathrm{I}$ & $\mathrm{I}$ & $\mathrm{F}$ & $\mathrm{U}$ & $\mathrm{VI}$ & $\mathrm{F}$ & $\mathrm{F}$ \\
\hline $\mathrm{e}_{3}$ & $\mathrm{I}$ & $\mathrm{I}$ & $\mathrm{VU}$ & $\mathrm{F}$ & $\mathrm{I}$ & $\mathrm{F}$ & $\mathrm{F}$ & $\mathrm{U}$ & $\mathrm{F}$ & $\mathrm{U}$ & $\mathrm{F}$ & $\mathrm{I}$ & $\mathrm{VI}$ & $\mathrm{U}$ & $\mathrm{F}$ & $\mathrm{I}$ \\
\hline
\end{tabular}

Table 3. Binary semantic forms of language evaluation results for equipment maintenance support evaluation indicators

\begin{tabular}{|c|c|c|c|c|c|c|c|c|c|c|c|c|c|c|c|c|}
\hline & $a_{1}$ & $a_{2}$ & $a_{3}$ & $a_{4}$ & $a_{5}$ & $a_{6}$ & $a_{7}$ & $a_{8}$ & $a_{9}$ & $a_{10}$ & $a_{11}$ & $a_{12}$ & $a_{13}$ & $a_{14}$ & $a_{15}$ & $a_{16}$ \\
\hline $\mathrm{e}_{1}$ & $\left(\mathrm{~S}_{1}, 0\right)$ & $\left(\mathrm{S}_{2}, 0\right)$ & $\left(\mathrm{S}_{2}, 0\right)$ & $\left(\mathrm{S}_{2}, 0\right)$ & $\left(\mathrm{S}_{0}, 0\right)$ & $\left(\mathrm{S}_{1}, 0\right)$ & $\left(\mathrm{S}_{2}, 0\right)$ & $\left(\mathrm{S}_{0}, 0\right)$ & $\left(\mathrm{S}_{0}, 0\right)$ & $\left(\mathrm{S}_{2}, 0\right)$ & $\left(\mathrm{S}_{0}, 0\right)$ & $\left(\mathrm{S}_{1}, 0\right)$ & $\left(\mathrm{S}_{1}, 0\right)$ & $\left(\mathrm{S}_{1}, 0\right)$ & $\left(\mathrm{S}_{1}, 0\right)$ & $\left(\mathrm{S}_{2}, 0\right)$ \\
\hline $\mathrm{e}_{2}$ & $\left(\mathrm{~S}_{0}, 0\right)$ & $\left(\mathrm{S}_{2}, 0\right)$ & $\left(\mathrm{S}_{0}, 0\right)$ & $\left(\mathrm{S}_{0}, 0\right)$ & $\left(\mathrm{S}_{1}, 0\right)$ & $\left(\mathrm{S}_{2}, 0\right)$ & $\left(\mathrm{S}_{0}, 0\right)$ & $\left(\mathrm{S}_{2}, 0\right)$ & $\left(\mathrm{S}_{1}, 0\right)$ & $\left(\mathrm{S}_{0}, 0\right)$ & $\left(\mathrm{S}_{2}, 0\right)$ & $\left(\mathrm{S}_{2}, 0\right)$ & $\left(\mathrm{S}_{0}, 0\right)$ & $\left(\mathrm{S}_{2}, 0\right)$ & $\left(\mathrm{S}_{2}, 0\right)$ & $\left(\mathrm{S}_{2}, 0\right)$ \\
\hline $\mathrm{e}_{3}$ & $\left(\mathrm{~S}_{4}, 0\right)$ & $\left(\mathrm{S}_{0}, 0\right)$ & $\left(\mathrm{S}_{3}, 0\right)$ & $\left(\mathrm{S}_{4}, 0\right)$ & $\left(\mathrm{S}_{4}, 0\right)$ & $\left(\mathrm{S}_{1}, 0\right)$ & $\left(\mathrm{S}_{4}, 0\right)$ & $\left(\mathrm{S}_{3}, 0\right)$ & $\left(\mathrm{S}_{3}, 0\right)$ & $\left(\mathrm{S}_{4}, 0\right)$ & $\left(\mathrm{S}_{0}, 0\right)$ & $\left(\mathrm{S}_{3}, 0\right)$ & $\left(\mathrm{S}_{3}, 0\right)$ & $\left(\mathrm{S}_{1}, 0\right)$ & $\left(\mathrm{S}_{1}, 0\right)$ & $\left(\mathrm{S}_{0}, 0\right)$ \\
\hline
\end{tabular}

Table 4. Binary semantic forms of evaluation results of equipment maintenance support evaluation index weights

\begin{tabular}{|c|c|c|c|c|c|c|c|c|c|c|c|c|c|c|c|c|}
\hline & $a_{1}$ & $a_{2}$ & $a_{3}$ & $a_{4}$ & $a_{5}$ & $a_{6}$ & $a_{7}$ & $a_{8}$ & $a_{9}$ & $a_{10}$ & $a_{11}$ & $a_{12}$ & $a_{13}$ & $a_{14}$ & $a_{15}$ & $a_{16}$ \\
\hline $\mathrm{e}_{1}$ & $\left(\mathrm{~S}_{2}, 0\right)$ & $\left(\mathrm{S}_{3}, 0\right)$ & $\left(\mathrm{S}_{3}, 0\right)$ & $\left(\mathrm{S}_{4}, 0\right)$ & $\left(\mathrm{S}_{3}, 0\right)$ & $\left(\mathrm{S}_{3}, 0\right)$ & $\left(\mathrm{S}_{4}, 0\right)$ & $\left(\mathrm{S}_{1}, 0\right)$ & $\left(\mathrm{S}_{3}, 0\right)$ & $\left(\mathrm{S}_{2}, 0\right)$ & $\left(\mathrm{S}_{3}, 0\right)$ & $\left(\mathrm{S}_{3}, 0\right)$ & $\left(\mathrm{S}_{2}, 0\right)$ & $\left(\mathrm{S}_{3}, 0\right)$ & $\left(\mathrm{S}_{3}, 0\right)$ & $\left(\mathrm{S}_{3}, 0\right)$ \\
\hline $\mathrm{e}_{2}$ & $\left(\mathrm{~S}_{4}, 0\right)$ & $\left(\mathrm{S}_{2}, 0\right)$ & $\left(\mathrm{S}_{1}, 0\right)$ & $\left(\mathrm{S}_{0}, 0\right)$ & $\left(\mathrm{S}_{2}, 0\right)$ & $\left(\mathrm{S}_{2}, 0\right)$ & $\left(\mathrm{S}_{3}, 0\right)$ & $\left(\mathrm{S}_{4}, 0\right)$ & $\left(\mathrm{S}_{3}, 0\right)$ & $\left(\mathrm{S}_{3}, 0\right)$ & $\left(\mathrm{S}_{3}, 0\right)$ & $\left(\mathrm{S}_{2}, 0\right)$ & $\left(\mathrm{S}_{1}, 0\right)$ & $\left(\mathrm{S}_{4}, 0\right)$ & $\left(\mathrm{S}_{2}, 0\right)$ & $\left(\mathrm{S}_{2}, 0\right)$ \\
\hline $\mathrm{e}_{3}$ & $\left(\mathrm{~S}_{3}, 0\right)$ & $\left(\mathrm{S}_{3}, 0\right)$ & $\left(\mathrm{S}_{0}, 0\right)$ & $\left(\mathrm{S}_{2}, 0\right)$ & $\left(\mathrm{S}_{3}, 0\right)$ & $\left(\mathrm{S}_{2}, 0\right)$ & $\left(\mathrm{S}_{2}, 0\right)$ & $\left(\mathrm{S}_{1}, 0\right)$ & $\left(\mathrm{S}_{2}, 0\right)$ & $\left(\mathrm{S}_{1}, 0\right)$ & $\left(\mathrm{S}_{2}, 0\right)$ & $\left(\mathrm{S}_{3}, 0\right)$ & $\left(\mathrm{S}_{4}, 0\right)$ & $\left(\mathrm{S}_{1}, 0\right)$ & $\left(\mathrm{S}_{2}, 0\right)$ & $\left(\mathrm{S}_{3}, 0\right)$ \\
\hline
\end{tabular}

(3) The binary semantic weights of different evaluation indicators are aggregated by formula (14), and then the arithmetic average binary semantic weights of the respective evaluation indexes are obtained: $\quad\left(\bar{w}_{1}, \bar{a}_{1}\right)=\left(s_{3}, 0\right), \quad\left(\bar{w}_{2}, \bar{a}_{2}\right)=\left(s_{3},-0.33\right), \quad\left(\bar{w}_{3}, \bar{a}_{3}\right)=\left(s_{1}, 0.33\right), \quad\left(\bar{w}_{4}, \bar{a}_{4}\right)=\left(s_{3}, 0\right) \quad, \quad\left(\bar{w}_{5}, \bar{a}_{5}\right)=\left(s_{3},-0.33\right)$, $\left(\bar{w}_{6}, \bar{a}_{6}\right)=\left(s_{2}, 0.33\right),\left(\bar{w}_{7}, \bar{a}_{7}\right)=\left(s_{3}, 0\right),\left(\bar{w}_{8}, \bar{a}_{8}\right)=\left(s_{2}, 0\right), \quad\left(\bar{w}_{9}, \bar{a}_{9}\right)=\left(s_{3},-0.33\right),\left(\bar{w}_{10}, \bar{a}_{10}\right)=\left(s_{2}, 0\right), \quad\left(\bar{w}_{11}, \bar{a}_{11}\right)=\left(s_{3},-0.33\right)$, $\left(\bar{w}_{12}, \bar{a}_{12}\right)=\left(s_{3}, 0\right),\left(\bar{w}_{13}, \bar{a}_{13}\right)=\left(s_{2}, 0.33\right),\left(\bar{w}_{14}, \bar{a}_{14}\right)=\left(s_{3},-0.33\right),\left(\bar{w}_{15}, \bar{a}_{15}\right)=\left(s_{2}, 0.33\right),\left(\bar{w}_{16}, \bar{a}_{16}\right)=\left(s_{3},-0.33\right)$.

(4) Using the formula (15) to process the results of the third step, and doing aggregation processing on the binary semantic evaluation results of different evaluation indicators, thus obtaining the weighted arithmetic mean binary semantic evaluation values of three experts: 


$$
\begin{aligned}
& R_{1}=\left(\bar{s}_{1}, \bar{a}_{1}\right)=\Delta\left(\frac{\Delta^{-1}\left(s_{1}, 0\right) \times \Delta^{-1}\left(s_{3}, 0\right)+\Delta^{-1}\left(s_{2}, 0\right) \times \Delta^{-1}\left(s_{3},-0.33\right)+\cdots+\Delta^{-1}\left(s_{2}, 0\right) \times \Delta^{-1}\left(s_{3},-0.33\right)}{\Delta^{-1}\left(s_{3}, 0\right)+\Delta^{-1}\left(s_{3},-0.33\right)+\cdots+\Delta^{-1}\left(s_{3},-0.33\right)}\right)=\left(\mathrm{s}_{1}, 0.12\right) \\
& R_{2}=\left(\bar{s}_{2}, \bar{a}_{2}\right)=\Delta\left(\frac{\Delta^{-1}\left(s_{0}, 0\right) \times \Delta^{-1}\left(s_{3}, 0\right)+\Delta^{-1}\left(s_{2}, 0\right) \times \Delta^{-1}\left(s_{3},-0.33\right)+\cdots+\Delta^{-1}\left(s_{2}, 0\right) \times \Delta^{-1}\left(s_{3},-0.33\right)}{\Delta^{-1}\left(s_{3}, 0\right)+\Delta^{-1}\left(s_{3},-0.33\right)+\cdots+\Delta^{-1}\left(s_{3},-0.33\right)}\right)=\left(\mathrm{s}_{1}, 0.14\right) \\
& R_{3}=\left(\bar{s}_{3}, \bar{a}_{3}\right)=\Delta\left(\frac{\Delta^{-1}\left(s_{4}, 0\right) \times \Delta^{-1}\left(s_{3}, 0\right)+\Delta^{-1}\left(s_{0}, 0\right) \times \Delta^{-1}\left(s_{3},-0.33\right)+\cdots+\Delta^{-1}\left(s_{0}, 0\right) \times \Delta^{-1}\left(s_{3},-0.33\right)}{\Delta^{-1}\left(s_{3}, 0\right)+\Delta^{-1}\left(s_{3},-0.33\right)+\cdots+\Delta^{-1}\left(s_{3},-0.33\right)}\right)=\left(\mathrm{s}_{2}, 0.23\right)
\end{aligned}
$$

(5) Using formula (14) to assemble, and assemble $R_{1}, R_{2} R_{3}$ to obtain the binary semantic comprehensive evaluation value of equipment maintenance support capability evaluation:

$$
(\bar{s}, \bar{a})=\Delta\left(\frac{\Delta^{-1}\left(s_{1}, 0.12\right)+\Delta^{-1}\left(s_{1}, 0.14\right)+\Delta^{-1}\left(s_{2}, 0.23\right)}{3}\right)=\left(\mathrm{s}_{1}, 0.47\right)
$$

It can be seen that the equipment maintenance support capability of the established maintenance and repair unit is at a relatively poor level, which reflects the overall level of the unit to be further improved. At the same time, it can be seen from the above that out of the 16 indicators, the scores of the maneuvering rate, equipment repair rate, maintenance qualification rate and personnel qualification rate are higher, while the motor consumption rate, maintenance safety rate and equipment matching rate score lower, in the future Unit construction should focus on strengthening. At the same time, in order to prove the validity of this method, this paper also adopts the analytic hierarchy process and BP neural network method to evaluate the unit's maintenance support ability. The simulation results using neural network simulation also have the poor guarantee ability, but not the size of an indicator is evaluated using the analytic hierarchy process. The evaluation results of the AHP and the binary semantics are shown in Table 5. The results of the analytic hierarchy process are basically similar to those of the dual semantics. The maneuverability rate, equipment repair rate, maintenance qualification rate, and staff qualification rate are also higher, while the motor consumption rate and social security reliability are ranked slightly differently. Research, comparative verification, and expert analysis have made the evaluation of binary semantics more accurate, demonstrating the effectiveness of the method.

Table 5. Comparison of assessment methods

\begin{tabular}{|c|c|c|c|c|c|c|c|c|}
\hline index & $a_{1}$ & $a_{2}$ & $a_{3}$ & $a_{4}$ & $a_{5}$ & $a_{6}$ & $a_{7}$ & $a_{8}$ \\
\hline Binary semantics & $(\mathrm{S} 3,0)$ & $(\mathrm{S} 3,-0.33)$ & $(\mathrm{S} 1,0.33)$ & $(\mathrm{S} 3,0)$ & $(\mathrm{S} 3,-0.33)$ & $(\mathrm{S} 2,0.33)$ & $(\mathrm{S} 3,0)$ & $(\mathrm{S} 2,0)$ \\
\hline AHP & 0.1071 & 0.0624 & 0.0312 & 0.0990 & 0.0559 & 0.0889 & 0.1087 & 0.0363 \\
\hline index & $a_{9}$ & $a_{10}$ & $a_{11}$ & $a_{12}$ & $a_{13}$ & $a_{14}$ & $a_{15}$ & $a_{16}$ \\
\hline Binary semantics & $(\mathrm{S} 3,-0.33)$ & $(\mathrm{S} 2,0)$ & $(\mathrm{S} 3,-0.33)$ & $(\mathrm{S} 3,0)$ & $(\mathrm{S} 2,0.33)$ & $(\mathrm{S} 3,-0.33)$ & $(\mathrm{S} 2,0.33)$ & $(\mathrm{S} 3,-0.33)$ \\
\hline AHP & 0.0312 & 0.0224 & 0.0562 & 0.1125 & 0.0757 & 0.0613 & 0.0301 & 0.0211 \\
\hline
\end{tabular}

\section{Concluding Remark}

Because the assessment indicators for equipment maintenance support capabilities are ambiguous, it is often difficult for experts to give more accurate assessment values. The use of binary semantic methods can fully eliminate the deficiencies of the indicators to evaluate the ambiguity, improve the accuracy of the assessment, and at the same time facilitate the experts to give assessment opinions, information can also effectively avoid the loss. On the basis of constructing the evaluation index system of equipment maintenance support capability, this paper proposes a method based on dual semantics to evaluate equipment maintenance support capability and conduct case analysis. Finally, comparing the binary semantics with analytic hierarchy process and BP neural network methods, the results show that this method can effectively evaluate the equipment maintenance support ability objectively and has certain reference value.

\section{References}

[1]. Yao Shilling, Dong Heaping, Zhou Hualon, Xu Change. Evaluation Index System for Basic Maintenance Support Capability of Armoured Equipment [J]. Sichuan Journal of Ordnance, 2009, 5(5):107-109. 
[2]. Wu Jian gang. Establishment of equipment maintenance support evaluation system [J]. Naval Equipment Maintenance, 2013, (3): 29-30.

[3]. Shad Xinyang, Zhou Shingle, Xu Yu. Construction of Evaluation Index System for Special Operations Ability Based on "Competency Model"[J]. Naval Academic Research, 2017, (1):47-49.

[4]. Zhao Xiao Hua, Liu Mingus, Wang Gushy, Zhu Aiming. Assessment of Support Ability of Sky wave Radar Equipment Maintenance Based on AHP [J]. Fire Control and Command Control,2014,39(12):145-148.

[5]. Zhang Lei, Wang Zedong, Hu Xiaoping. A Simulation-Based Method for Assessing Equipment Maintenance Support Capability [J]. Fire Control and Command Control, 2014, 39(4):106-109.

[6]. Hu Zhen qi, Xiao Wu. Optimization of concurrent mining and reclamation plans for single coal seam: a case study in northern Anhui, china [J]. Environmental Earth Sciences, 2013(5). 